\title{
Polarization Dependence of Surface Enhanced Raman Scattering on a Single Dielectric Nanowire
}

\author{
Hua Qi, R. W. Rendell, O. J. Glembocki, and S. M. Prokes \\ Electronics Science and Technology Division, Naval Research Laboratory, Washington, DC 20375, USA \\ Correspondence should be addressed to Hua Qi, qhqihua@yahoo.com \\ Received 15 June 2012; Accepted 8 September 2012 \\ Academic Editor: Steve F. A. Acquah \\ Copyright () 2012 Hua Qi et al. This is an open access article distributed under the Creative Commons Attribution License, which \\ permits unrestricted use, distribution, and reproduction in any medium, provided the original work is properly cited.
}

Our measurements of surface enhanced Raman scattering (SERS) on $\mathrm{Ga}_{2} \mathrm{O}_{3}$ dielectric nanowires (NWs) core/silver composites indicate that the SERS enhancement is highly dependent on the polarization direction of the incident laser light. The polarization dependence of the SERS signal with respect to the direction of a single NW was studied by changing the incident light angle. Further investigations demonstrate that the SERS intensity is not only dependent on the direction and wavelength of the incident light, but also on the species of the SERS active molecule. The largest signals were observed on an NW when the incident $514.5 \mathrm{~nm}$ light was polarized perpendicular to the length of the NW, while the opposite phenomenon was observed at the wavelength of $785 \mathrm{~nm}$. Our theoretical simulations of the polarization dependence at $514.5 \mathrm{~nm}$ and $785 \mathrm{~nm}$ are in good agreement with the experimental results.

\section{Introduction}

Surface enhanced Raman scattering (SERS) has been regarded as a unique technique to detect trace levels of chemical compounds, since the vibrational information is very specific to the bonds in the molecules. In recent years, there has been significant interest in exploring various nanostructures as SERS substrates to optimize the electromagnetic field enhancement and significantly improve sensitivity. In the case of nonspherical nanoparticles it has been shown that the SERS enhancement is strongly dependent on the direction of polarization of the exciting incident light [1-12]. Of particular interest are cylindrical geometries because the enhancement is a function of the aspect ratio. In addition, in the case of very long $\mathrm{Ag}$ nanowires it has been shown that surface plasmons can be launched at one end of the nanowires and travel to the other end. Recently there has been interest in metal coated dielectric core nanowires because they form plasmonic shells $[13,14]$. These geometries can form the basis of plasmonically modulated photonic devices. It is well known that the local electric field in plasmonic coupling of closely spaced particles, usually called "hot spots," can be orders of magnitude stronger than those on individual particles. The importance of "hot spots" in SERS process has been widely discussed [15-21].

In this work, a highly effective SERS composite of dielectric $\mathrm{Ga}_{2} \mathrm{O}_{3}$ NWs core/silver was employed to investigate the SERS intensity dependence on the laser polarization. Experimental results show that both variable wavelength and angle of the incident light play very important roles on the resulting SERS intensity. Our theoretical simulations indicated that the maximum SERS enhancement could be obtained when the polarization is perpendicular to the NW length at $514.5 \mathrm{~nm}$ excitation, while the opposite phenomena should be expected at a laser wavelength of $785 \mathrm{~nm}$. All of these expectations are in good agreement with our experimental observations. In addition, further experiment shows that the orientation of self-assembled monolayer of active SERS molecules on the NWs may affect the SERS enhancement as well.

\section{Experimental Details}

Random $\mathrm{Ga}_{2} \mathrm{O}_{3}$ NWs were grown via the vapor-liquid-solid (VLS) growth mechanism [22]. The gallium metal (99.995\% pure) used as a source was placed 6 inches upstream from 
the Si (100) substrate, which had a $20 \mathrm{~nm}$ gold film. The furnace was heated to $900^{\circ} \mathrm{C}$ while flowing simultaneously a mixture of argon and oxygen gases in a ratio of $6: 1$ through the tube. To study the SERS behavior of a single NW, the $\mathrm{Ga}_{2} \mathrm{O}_{3}$ NWs as grown on a Si substrate were sonicated off in methanol, dropped and dried on a bare silicon surface for further SERS study.

The discrete single NWs were covered with a layer of silver produced by an electroless (EL) plating approach for the SERS behavior study, which has been described in our previous report $[13,14]$. Briefly, the silver ion in Tollen reagent was reduced to neutral Ag and then homogenously deposited on the NWs surface. The morphology of the silver on the NW surface is directly related to the chemical concentration and reaction time. Usually, short reaction time of the silver electroless plating process results in closely spaced but separate nanoparticles, while longer times lead to linking up of the islands into a rough layer.

The SERS line maps were carried out using a confocal $\mu$-Raman system which consisted of a Mitutoyo Microscope and an Ocean Optics QE65000 spectrometer equipped with a thermoelectrically cooled CCD. The $514.5 \mathrm{~nm}$ line of an $\mathrm{Ar}^{+}$ ion laser was used as the excitation source. The microscope utilized a 100x 0.7 NA objective for focusing the laser light and was coupled to the spectrometer through a fiber optic cable. The maps were collected with low laser power of $0.75 \mathrm{~mW}$ at the sample. This was done to prevent desorption and damage to the benzenethiol and to prevent alterations to the Ag layer. The SERS intensity dependence on variable polarization angles was performed using a Delta $\mathrm{Nu}$ system which consists of an Olympus Microscope and a Raman spectrometer equipped with a thermoelectrically cooled CCD. The $785 \mathrm{~nm}$ line of Ti: Sapphire laser was used as the excitation source to detect the SERS strength dependence on a single NW/silver composite. The microscope utilized a 50x $0.75 \mathrm{NA}$ objective for focusing the laser light. The spectra were collected with a laser power of $3 \mathrm{~mW}$ at the sample.

The full Maxwell's equations are solved numerically using the finite elements method (FEM) through the RF module of the COMSOL Multiphysics finite-elements package [23]. We used FEM simulations because they are more appropriate for the complex geometries of nanowires placed on a substrate [24]. FEM approaches have been shown to agree well with finite difference time domain simulations [24, 25]. In addition, the FEM technique implemented via COMSOL has been shown to be capable of accurate simulation for nanoshell structures on substrates if sufficiently accurate meshing is used and appropriate absorbing perfectly matched layers PML are used on the boundaries [26-28]. This approach allows us to treat the scattering problem by bringing in planes waves of a specific polarization and then calculating the scattered fields. As another test of the numerical simulations, we find excellent agreement between the COMSOL results with the analytical Mie solutions for scattering from an Ag sphere [24, 27]. The modeling of the nanowires in air above an Si substrate requires the construction of separate PML boundaries for both the Si and air regions. The dielectric constants of each PML match its adjoining region, either $\mathrm{Si}$ or air. To avoid artifacts due to scattering at the boundaries between the Si and air regions of the PML's, the scattering at the air-Si interface is incorporated into the incident-polarized plane wave by means of analytical functions for the Fresnel coefficients. The resulting incident field is thus an exact solution in the absence of the nanowire. The numerical finite element solution for the scattered field is then calculated in the presence of the nanowire. This requires sufficient meshing to adequately resolve both the nanowires with shells and the PML boundaries [27, 28].

\section{Results and Discussion}

To demonstrate the surface enhanced Raman scattering (SERS) polarization dependence on the direction of the nanowires (NWs), we carried out the mapping investigations on NW/Ag composite on the same NW using a $514.5 \mathrm{~nm}$ excitation with parallel and perpendicular laser polarization, respectively. The $\mathrm{Ga}_{2} \mathrm{O}_{3}$ NWs, used in all experiments, were grown via the vapor-liquid-solid (VLS) growth mechanism. Empirically, the length and diameter of $\mathrm{Ga}_{2} \mathrm{O}_{3} \mathrm{NWs}$ depended on the growth time and gas flow rate, as well as the catalyst size. In our growth, the diameters varied from $50 \mathrm{~nm}$ to $300 \mathrm{~nm}$, and the length was usually greater than 10 microns. Energy dispersive X-ray (EDX) analysis showed that the chemical composition of the NWs was stoichiometric $\mathrm{Ga}_{2} \mathrm{O}_{3}$. Benzenethiol (BT) was used as the SERS active molecules.

The intensity dependence maps of the $1576 \mathrm{~cm}^{-1}$ BT SERS band on incident laser polarization are shown in Figures 1(a) and 1(b), by which the polarization dependence can be easily visualized. Both of the images were mapped at the same region of the sample by rotating the sample. Figure 1(c) shows the corresponding microscope image of the SERS mapping area. From the maps, it is obvious that the signal strength is maximized when the laser is perpendicular to the NW.

To verify our experimental observation, we performed the simulations using the RF module of COMSOL Multiphysics, which provides a finite-element solution of Maxwell's equations. Figures 2(a) and 2(b) show the simulated SERS maps of the NW with length of $500 \mathrm{~nm}$ at a laser wavelength of $514.5 \mathrm{~nm}$, indicating that the strongest SERS signal occurs when the polarization is perpendicular to the NW longitude, which clearly confirmed the incident laser polarization effects on the SERS strength, as we obtained in experiments shown in Figure 1. It is noted in the maps that the plasmon oscillations are clearly observed along the NW when the polarization is parallel to the NW, while the transverse modes have excitations along the radial direction and hence much shorter period and uniform in NW's longitudinal direction.

Furthermore, more comprehensive simulations were carried out in the range of wavelength from $400 \mathrm{~nm}$ to $900 \mathrm{~nm}$, as shown in Figure 3, which includes the wavelength of $514.5 \mathrm{~nm}$ used in the mapping experiments and simulations above. By comparison of Figures 3(a) and 3(b), it is obvious that, in the case of parallel, the SERS enhancement factor is $10^{6} \sim 10^{7}$, while the perpendicular case shows 


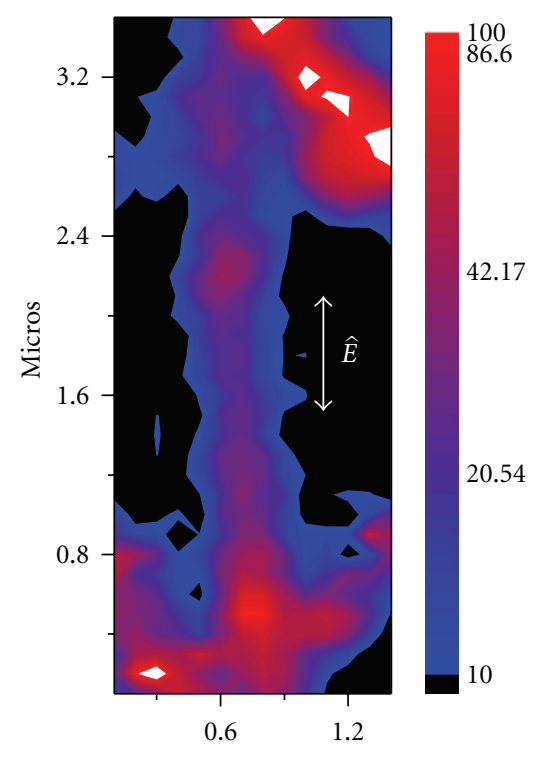

(a)

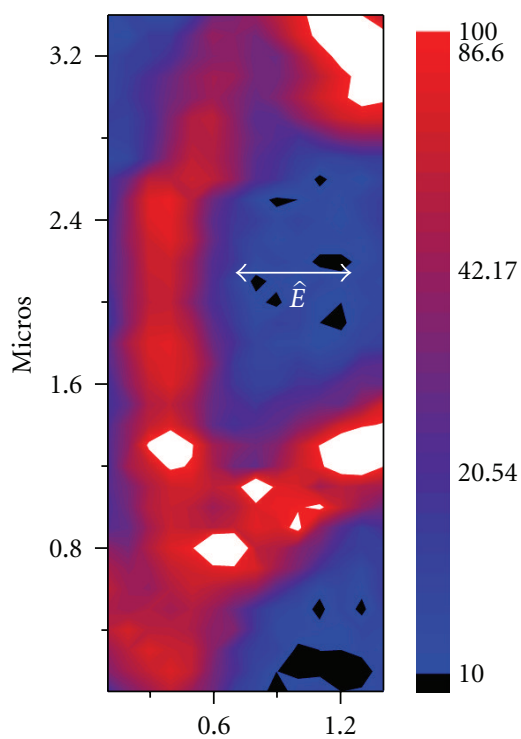

(b)

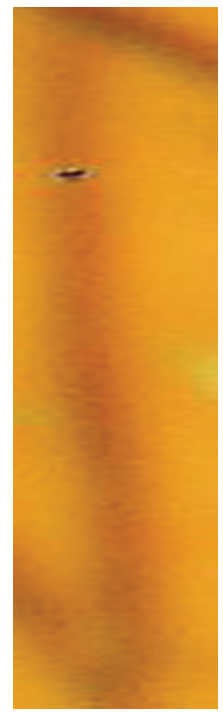

(c)
FIGURE 1: Experimental maps of the $1576 \mathrm{~cm}^{-1}$ BT SERS band of NW/Ag composite using a laser with $514.5 \mathrm{~nm}$ wavelength at the same NW, and the sample was rotated for parallel/perpendicular polarization mapping; the polarization is parallel (a) and perpendicular (b) to the NW. (c) Corresponding microscope image of the SERS mapping region of (a) and (b).

an enhancement factor of $10^{8} \sim 10^{9}$, clearly indicating that the NW/Ag composites show stronger SERS enhancement when the laser polarization is perpendicular to the NW direction at $514.5 \mathrm{~nm}$, which is in good agreement with the experimental mapping images of Figure 1 and the theoretical simulation mapping results of Figure 2.

From Figures 3(a) and 3(b), it is expected, at the wavelength of $785 \mathrm{~nm}$ (red laser), that the strongest SERS signals should be observed when the laser polarization parallelized to the NW longitude direction, which is completely opposite

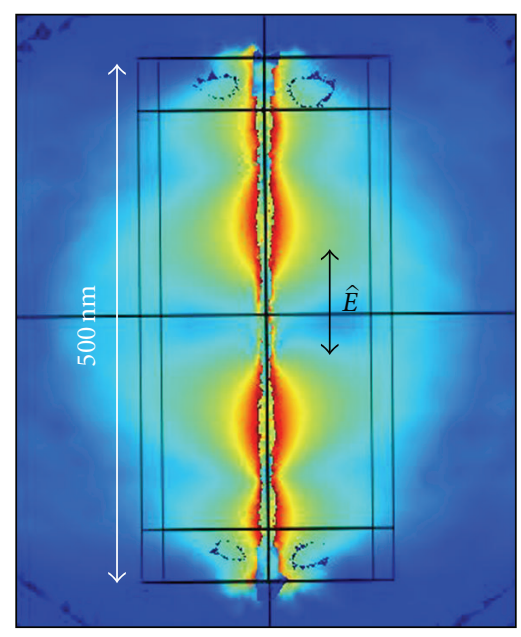

(a)

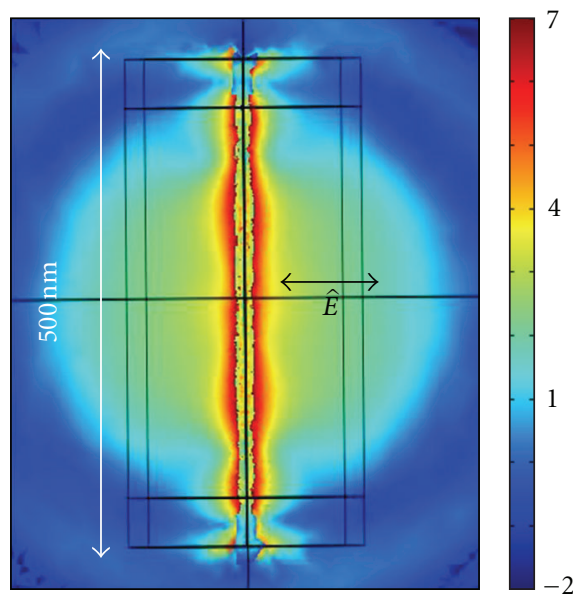

(b)

FIGURE 2: Log plots of SERS enhancement simulated at a wavelength of $514.5 \mathrm{~nm}$ when the NW direction is parallel (a) and perpendicular (b) to the polarization.

to the observations at the excitation of $514.5 \mathrm{~nm}$. Hence, we performed further investigations of SERS enhancements dependence on the incident angles using incident laser of $785 \mathrm{~nm}$ and $514.5 \mathrm{~nm}$. In the process of measurements, data were collected at three different angles, including $0^{\circ}$, $45^{\circ}$, and $90^{\circ}$, respectively. The representative BT SERS line of $1576 \mathrm{~cm}^{-1}$ at different angles and laser wavelengths are shown in Figure 4. The red and green lines are roughly linear fitting of the experimental data obtained at three angles, which clearly indicates the trends of SERS enhancement dependence on the incident laser angles and the different wavelengths. It is noted that the data were normalized to the maximum intensity.

More detailed simulations of the SERS polarization dependence of the NW core/Ag composites for the incident wavelength of $785 \mathrm{~nm}$ and $514.5 \mathrm{~nm}$ are shown in Figures 5(a) and 5(b), respectively, which provide a continuous SERS enhancement trend from $0^{\circ}$ to $90^{\circ}$. As can be seen in the calculation, the largest SERS enhancement is completely flipped 


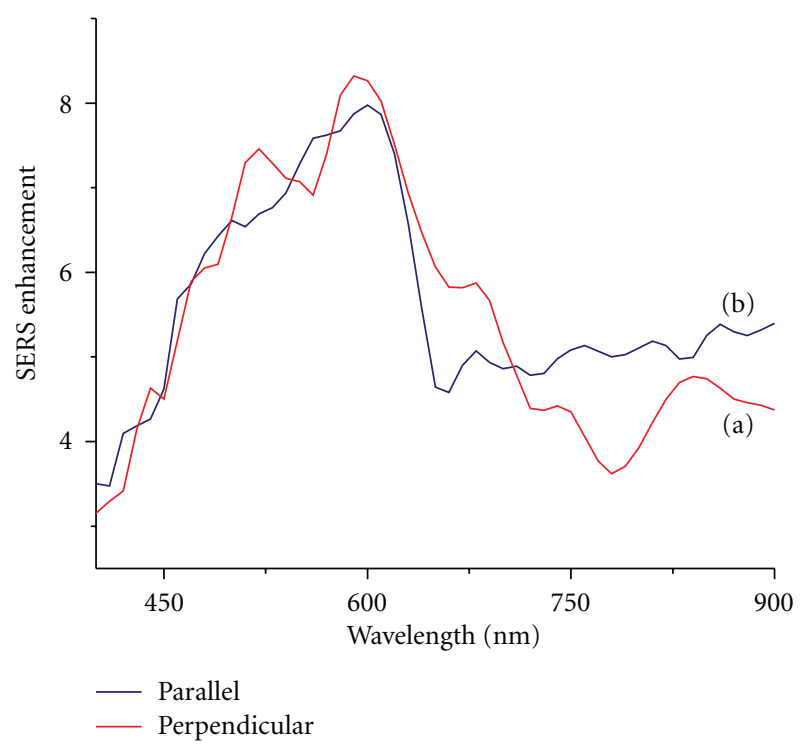

FIGURE 3: Simulations of SERS enhancements in the range of wavelength from $400 \mathrm{~nm}$ to $900 \mathrm{~nm}$. The NW direction is parallel (red) and perpendicular (blue) to the polarization.

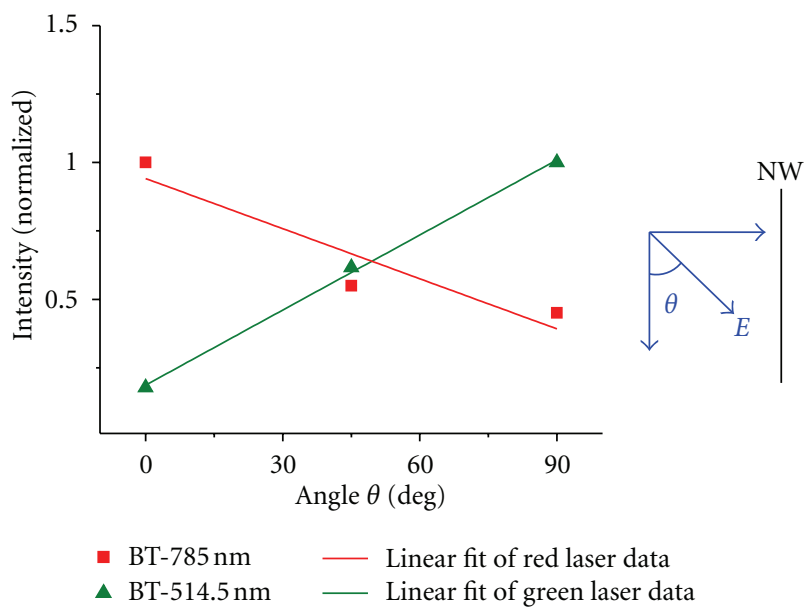

Figure 4: Experimental polarization angle dependence of surface enhanced Raman scattering (SERS) on nanowire at the wavelength of $\lambda=785 \mathrm{~nm}$ (red squares and its linear fitting line) and $\lambda=$ $514.5 \mathrm{~nm}$ (green triangles and its linear fitting line) the data were normalized to the maximum intensity.

at the excitation wavelength of $785 \mathrm{~nm}$ and $514.5 \mathrm{~nm}$, which matches very well with our experimental observations as shown in Figure 4. For SERS, the Raman intensity generally increases by a factor of $E^{4}$, where the first two powers of the enhancement are due to the local electromagnetic field which in the present case is predominantly due to the contact between the nanowire and the Si substrate. The second two powers of the enhancement factor are the Raman emission enhancement. As a result of these two contributions to the SERS enhancement, it has been argued [9] that this should lead to a simple $\sin ^{2}(\theta)$ polarization dependence for a nanowire. If $\theta$ is the polarization angle of the incident light with respect to the direction along the length of the nanowire, then the local electric field is $\left|E_{\mathrm{loc}}(\omega, \theta)\right|=$ $\left|E_{\max }(\omega)\right| \sin (\theta)$ where $E_{\max }$ is the maximum of the local electric field. However, the direction of the induced electric field between the nanowire and the substrate is always in the direction across the nanowire-substrate contact $(\theta=\pi / 2)$ and independent of the incident polarization. Thus the total SERS enhancement is expected to be proportional to

$$
\begin{aligned}
G & =\left|\frac{E_{\max }\left(\omega_{L}\right)}{E_{0}\left(\omega_{L}\right)}\right|^{2}\left|\frac{E_{\max }\left(\omega_{R}\right)}{E_{0}\left(\omega_{R}\right)}\right|^{2} \sin ^{2}\left(\theta+\varphi_{\text {phase }}\right) \\
& =\left|\frac{E_{\max }}{E_{0}}\right|^{4} \sin ^{2}\left(\theta+\varphi_{\text {phase }}\right),
\end{aligned}
$$

where generally a phase shift phase is also expected due to the fact that metallic nanowires on substrates which are tens of microns in length can function as Fabry-Perot cavities resulting in geometry specific standing plasmon waves $[26,29,30]$. Polarization dependences as in (1) have been previously observed $[9,30]$. This is also consistent with the simulation results in Figure 5 where the difference between (a) and (b) can be described in terms of a different phase shift occurring at each excitation wavelength. This can be expected due to the observations that in the Fabry-Perot behavior of nanowires on substrates $[26,29]$, the surface plasmon wavelength has been found to differ from the excitation wavelength (e.g., between $400-600 \mathrm{~nm}$ for excitation at $785 \mathrm{~nm}[26,29])$ and is geometry dependent. As a result, the plasmon standing waves will generally occur with different relative phase shifts and the maximum enhancement may occur at a different polarization angle depending on the excitation wavelength. This has been observed for the first time in the present work and the result is consistent with previous observations of metallic nanowires on substrates $[26,29,30]$.

From the comprehensive simulation results of Figure 3, we can see that the polarization angle dependence of the wire at $633 \mathrm{~nm}$ would exhibit similar behavior to that shown for the $514 \mathrm{~nm}$ in Figure 5(b), but the SERS enhancement in the $90^{\circ}$ case shows stronger enhancements and more extended fields compared to the $514 \mathrm{~nm}$ case shown in Figure 2(a). For the $0^{\circ}$ case, the SERS enhancement would be predicted to be weaker at $633 \mathrm{~nm}$ compared to the $514 \mathrm{~nm}$ case. This is shown in Figure 6.

In addition, the NW/Ag composites SERS dependence on the polarization was experimentally investigated using a laser excitation of $785 \mathrm{~nm}$ to investigate the incident angle effects on the SERS enhancement in detail. Figures 7 (a) and $7(\mathrm{~b})$ show the high resolution SEM image of an isolated bare NW and NW/Ag composites. As shown in Figure 7(b), a rough layer of silver was clearly plated on the NW surface, which is produced by an electroless (EL) approach $[13,14]$. From the SEM image, the silver nanoparticles display 3D islands with a diameter range of $20 \mathrm{~nm}$. The space between the particles is less than $10-20 \mathrm{~nm}$, which leads to the strong plasmonic oscillation due to coupling of individual nanoparticles/island formed on NWs, as we reported previously [13]. The SERS spectra were carried out by changing the angle $\theta$ in $15^{\circ}$ increments between the electric field of the incident light and the axis parallel to the NW length, as shown in 


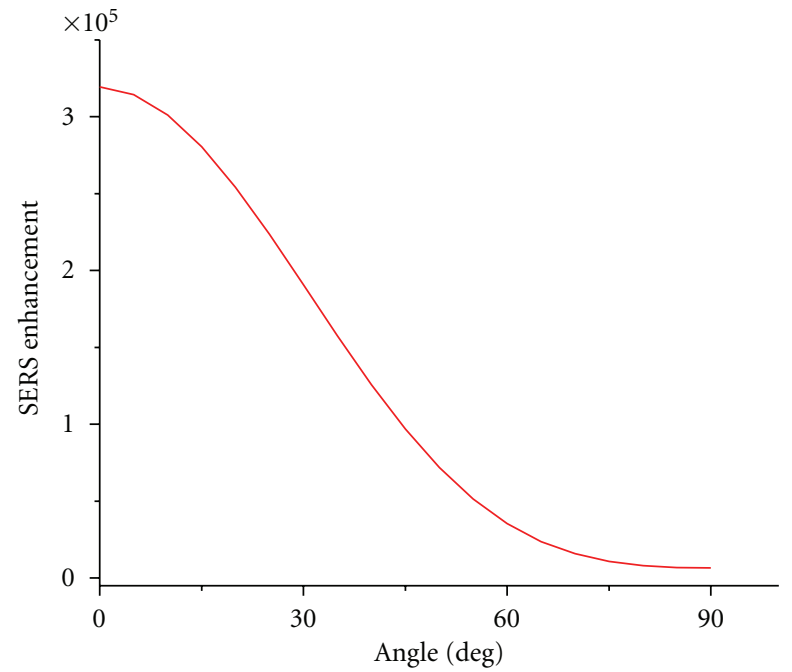

(a)

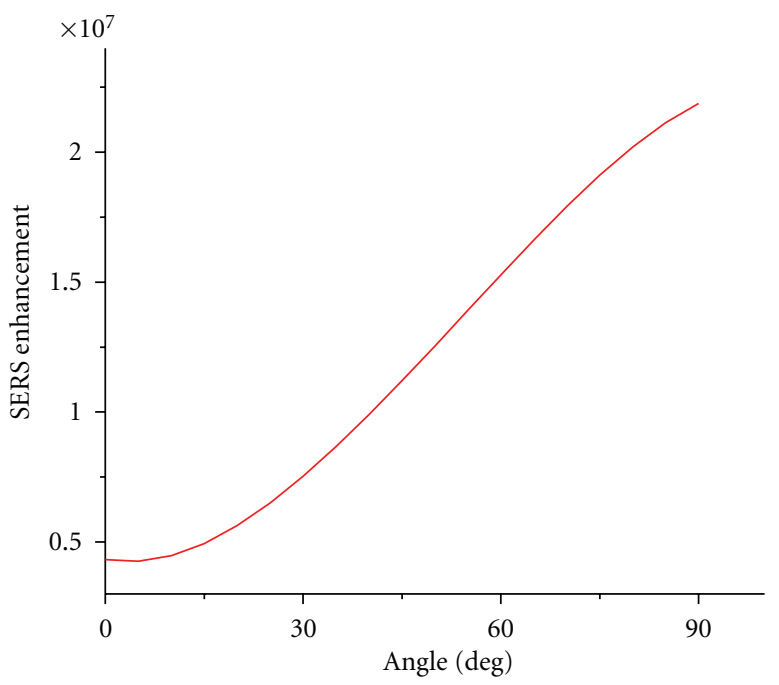

(b)

FIGURE 5: Simulation of polarization angle dependence of surface enhanced Raman scattering (SERS) averaged over nanowire surface excluding the wire ends for (a) $\lambda=785 \mathrm{~nm}$ and (b) $\lambda=514.5 \mathrm{~nm}$.

the sketch of Figure 7(b). To minimize the time-dependence degradation influence of the SERS signal intensity during the measurements, two cycles of SERS measurements, from $0^{\circ}$ to $90^{\circ}$, then back to $0^{\circ}$, were performed on a single NW. The data obtained from those two cycles were averaged for the spectra plots of each angle.

Figure 7(c) shows a series of SERS spectra as a function of the incident light polarization angles from $0^{\circ}$ to $90^{\circ}$. Here benzenethiol (BT) molecules were used as SERS active molecules. The major Raman peaks at 1001, 1023, 1073, and $1574 \mathrm{~cm}^{-1}$ can be assigned to symmetric ring breathing, inplane phenyl ring bending, in plane $\mathrm{C}-\mathrm{H}$ bending, and in plane $\mathrm{C}-\mathrm{C}$ stretching of the phenyl ring from $\mathrm{BT}$, respectively, which is in good agreement with those reported previously [31-36]. It is clear that the SERS signal displayed strong dependence on the polarization direction of the incident

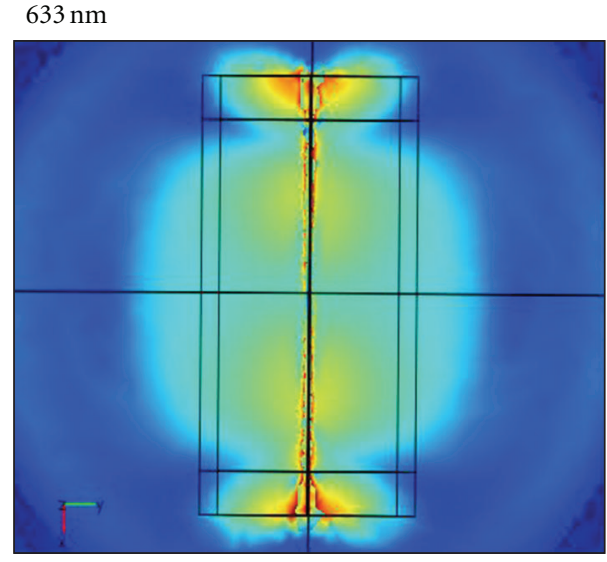

$0^{\circ}$

(a)

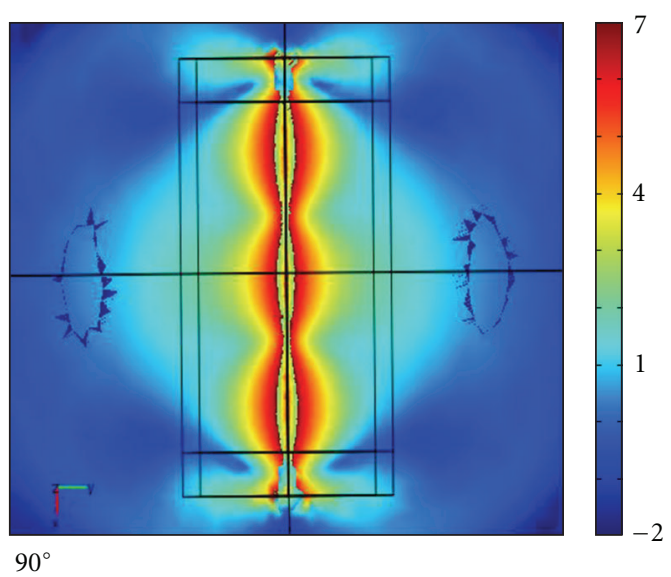

(b)

FIGURE 6: Log plots of SERS enhancement simulated at a wavelength of $633 \mathrm{~nm}$ when the NW direction is parallel (a) and perpendicular (b) to the polarization.

laser on a single NW, and the strongest SERS intensity was observed when the polarization is parallel to the NW length at an angle of $0^{\circ}$.

A plot of the normalized intensity data of the representative BT SERS band as a function of polarization angle is shown in Figure 7(d) (red curve), by which the polarization dependence can be easily visualized. It is obvious that the strength of SERS signals is maximized at $0^{\circ}$, which is different from the SERS strength dependence on laser polarization reported previously on pure silver or gold NWs with sparse noble metal particles $[8,9]$. The latter reported that the maximum of the SERS enhancement was observed when the polarization was perpendicular to the metal NWs. The difference may be attributed to the fact that the dielectric $\mathrm{Ga}_{2} \mathrm{O}_{3}$ NWs SERS substrate and the uniform coverage of silver particles layer play important roles in this polarization dependence process. That is to say the NWs SERS behavior is also related to the structure and composites of the SERS substrate. 


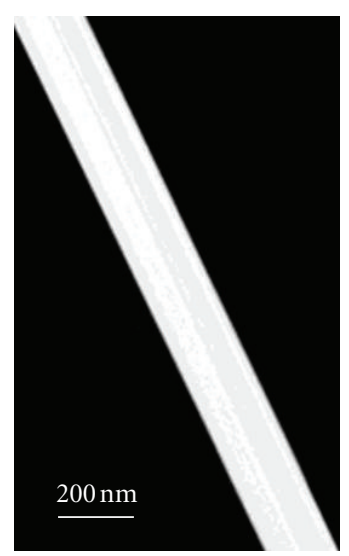

(a)

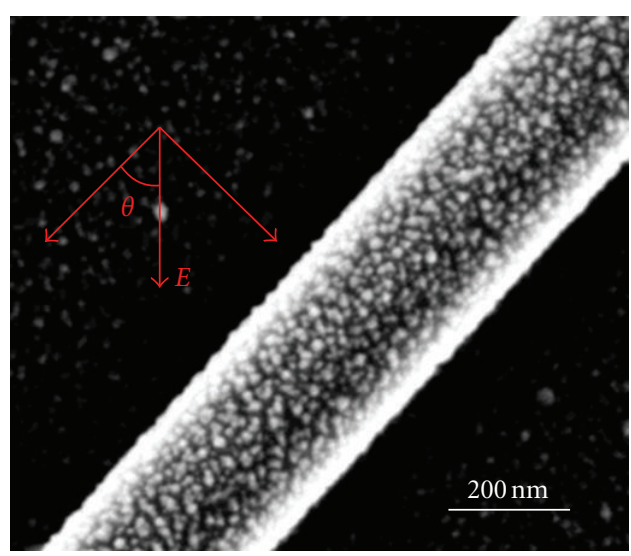

(b)

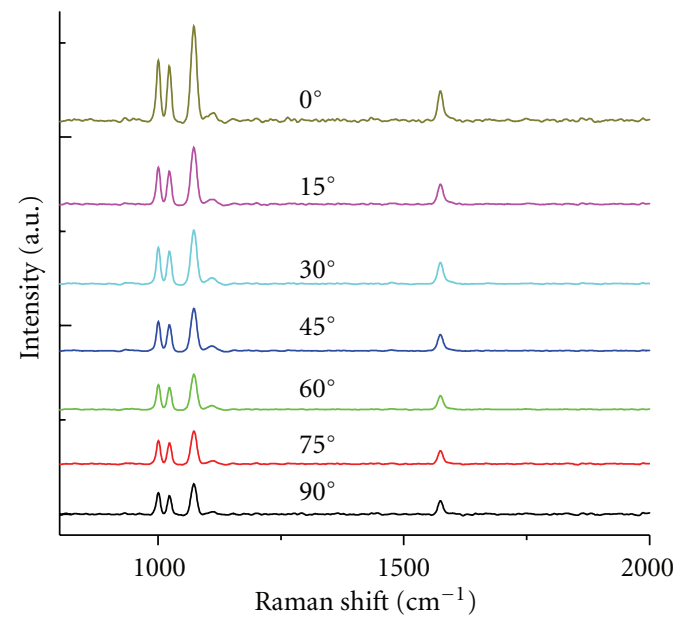

(c)

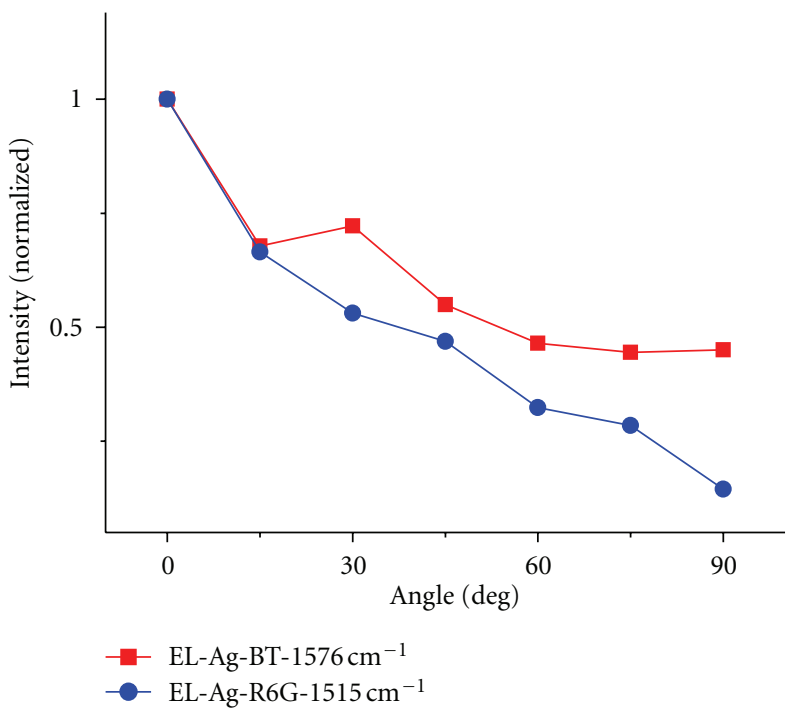

(d)

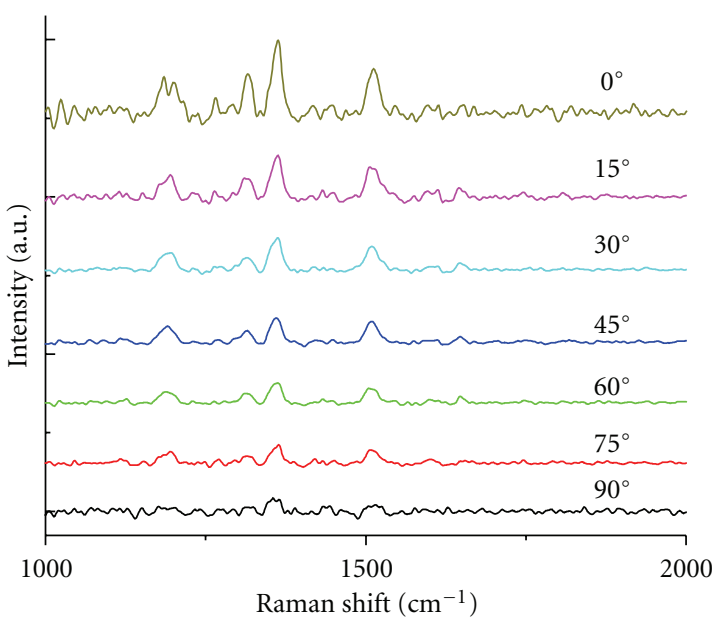

(e)

FIGURE 7: SEM image of a bare (a) and an electroless silver plated (b) NW. Inset of (b): the sketch indicates the laser polarization direction. (c) SERS dependence of a single NW/Ag composite on the laser polarization angles for Benzenethiol (BT) molecules at a wavelength of $785 \mathrm{~nm}$. (d) Plot of intensity of SERS representative lines of BT (red) and rhodamine 6G (R6G) (blue) versus angles and the data were normalized to the maximum intensity. (e) SERS dependence of a single NW/Ag composite on the laser polarization angles for R6G molecules at a wavelength of $785 \mathrm{~nm}$.

Additionally, a bump was observed at the angle of around $30^{\circ}$ for BT molecules as shown in Figure $7(\mathrm{~d})$ (red curve). A possible explanation to this observed bump around $30^{\circ}$ is that the orientation of the attached active molecules may have effect on this polarization dependence behavior. It is well known that the benzene rings of BT could easily form a self-assembled monolayer on a silver surface. In our study, the dielectric core was wrapped with a layer of silver, which provides a favorable condition for the formation of the ordered self-assembled monolayer (SAM). However, numerous reports in the literatures indicate that the orientation of the phenyl ring plane vary from perpendicular to flat from the substrate surface [37-45]. The SAM orientation of BT may affect the SERS enhancement factor when the incident light angle is variable. Here, as shown in the Figure 8(a), we assumed that the BT SAM was deduced to be tilted about $60^{\circ}$ from the substrate surface, which is similar to the research results obtained by Szafranski et al. [37]. Hence the abrupt increase of SERS signals at the angle of around $30^{\circ}$ could be attributed to the perpendicularity between the laser polarization direction and the phenyl ring plane. This is consistent with the electrostatic model interpretation of Gunnarsson et al. [46] for the relative SERS intensities of rhodamine $6 \mathrm{G}$ and thiophenol in terms of the orientation of the phenol ring out from the surface. This observation implies that the SERS signal strength highly depends on the molecules packing structure on the active substrate, which may provide a useful way to investigate the orientation of self-assembly molecular monolayer on surface via SERS technique. 

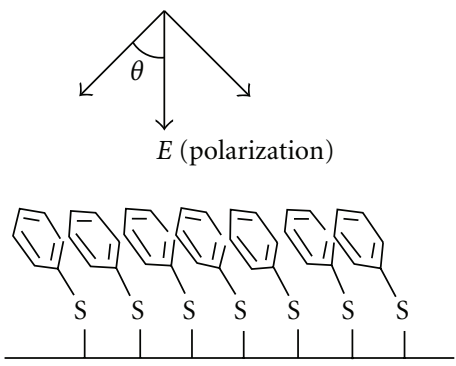

NW with silver layer

(a)<smiles>CCN=c1cc2oc3cc(NCC)c(C)cc3c(-c3ccccc3C(=O)OCC)c-2cc1C</smiles>

(b)

FIGURE 8: (a) A scheme of BT molecules orientation on the substrate and their interaction with polarized laser. (b) The structure of R6G.

To eliminate the effect of the SAM on the polarization behavior, and to investigate the pure polarization dependence on the NW direction, a random physically adsorbed layer of rhodamine $6 \mathrm{G}$ (R6G) was employed to study the SERS dependence on a single $\mathrm{Ga}_{2} \mathrm{O}_{3}$ NW. Figure $7(\mathrm{e})$ shows a series of SERS spectra of R6G with variable angles. Since R6G was attached randomly on an NW surface, this could effectively remove the polarization effect caused by the ordered molecular orientation. Hence the SERS polarization dependence behavior would completely come from the NW/Ag composite. Representative SERS line of R6G versus angle was plotted in Figure $7(\mathrm{~d})$, which clearly show that the signal intensity of R6G increases with a decrease of the incident light angle. The signal is maximized when the polarization direction of the light is parallel to the NW direction $\left(\theta=0^{\circ}\right)$, which is in good agreement with the observation of BT molecule at the same incident wavelength of $785 \mathrm{~nm}$.

It is observed that R6G random molecules layer shows a stronger polarization effect than BT ordered layer, which is opposite to what one would expect. However if the size of the molecules and the roughness of the silver particles on NWs are taken into account, the fact should be easier to understand. As shown in Figure 8, it is clear to see that the area of R6G is much larger than BT. This means that, per unit, the number of R6G molecules is much smaller than BT, which results in the ordered fact of benzene rings in the case of R6G, somehow. Additionally the silver particles on NWs are separated island, not perfectly smooth surface, which may cause the disorder pack of the BT molecules, and reduce the enhancement dependence on the polarization. Based on these facts, it is possible and reasonable that R6G layer shows a stronger polarization effect.
It has been reported that the bare single-crystalline silver NW showed SERS enhancement only when the laser polarization direction was perpendicular to the long axis of the NW [47]. In the case of the dielectric $\mathrm{Ga}_{2} \mathrm{O}_{3} \mathrm{NW}$ core/silver layer composites, we observed SERS enhancements for both perpendicular and parallel polarization directions. This can also be attributed to the structure difference of the SERS active substrate. Some calculations indicated that the maximum enhancement of the plasmon resonances in noble metal spheroids arises from the region of the highest curvature [48-50]. Recently Hill et al. reported the high efficacy of gold-nanoparticle- (NP-) gold film system in generating large field enhancements due to the extremely close distance between the NP and the film, clearly demonstrating the importance of the substrate gap for SERS enhancement [51]. We have also demonstrated that there is a significant interaction between the spherical silver NPs on the ZnO NW due the closely spaced NPs, and the islands also interact with the dielectric NW substrate, further increasing the enhancement [13]. In addition, the SERS enhancement dependence on the polarization direction was observed for all bands at the excitation source of $785 \mathrm{~nm}$ and $514.5 \mathrm{~nm}$. In recent years, a significant amount of work has been published on SERS, some of which has been on nanostructures, such as $1 \mathrm{D}$ nanowires $[52,53]$. However, there are no reports of results such as those that we are presenting in this work. Thus all of these studies deepen the understanding of the strong SERS enhancement using dielectric NW core/silver layer composites and the underlying plasmonic behavior, which is critical to the development of sensor technology that can eventually be used for trace chemical, biological, and explosive sensing.

\section{Conclusions}

We have demonstrated the strong SERS dependence on the polarization direction of the incident light by investigating dielectric $\mathrm{Ga}_{2} \mathrm{O}_{3} \mathrm{NW}$ core/silver composites, which showed that the SERS enhancement not only relies on the polarization direction of light with respect to the NW, but also on the wavelength of the incident laser and the orientation of SERS active molecules. Specifically, the strongest intensity of the SERS enhancement is obtained when the polarization of the incident light is parallel to the NW length at the incident wavelength of $785 \mathrm{~nm}$, while the signal is maximized when the polarization is perpendicular to the NW length at $514.5 \mathrm{~nm}$, which were confirmed by both experimental and theoretical simulation results. Therefore the SERS enhancement can be maximized by optimizing the direction of the NW, the incident angle of the laser polarization, the orientation of the molecules, and the wavelength of the laser.

\section{Acknowledgments}

This work was partially supported by the Office of Naval Research (ONR) and Nanoscience Institute (NSI) of the US Naval Research Laboratory. The authors thank Dr. D. A. Alexson for his help during some data collections. 


\section{References}

[1] H. Xux and M. Käll, "Polarization-dependent surface-enhanced raman spectroscopy of isolated silver nanoaggregates," ChemPhysChem, vol. 4, no. 9, pp. 1001-1005, 2003.

[2] J. P. Kottmann and O. J. F. Martin, "Plasmon resonant coupling in metallic nanowires," Optics Express, vol. 8, no. 12, pp. 655-663, 2001.

[3] S. A. Maier, P. G. Kik, H. A. Atwater et al., "Local detection of electromagnetic energy transport below the diffraction limit in metal nanoparticle plasmon waveguides," Nature Materials, vol. 2, no. 4, pp. 229-232, 2003.

[4] T. Dadosh, J. Sperling, G. W. Bryant et al., "Plasmonic control of the shape of the raman spectrum of a single molecule in a silver nanoparticle dimer," ACS Nano, vol. 3, no. 7, pp. 19881994, 2009.

[5] I. K. J. Kretzers, R. J. Parker, R. V. Olkhov, and A. M. Shaw, "Aggregation kinetics of gold nanoparticles at the silica-water interface," Journal of Physical Chemistry C, vol. 113, no. 14, pp. 5514-5519, 2009.

[6] Y. Sun, G. Wei, Y. Song et al., "Type I collagen-templated assembly of silver nanoparticles and their application in surfaceenhanced Raman scattering," Nanotechnology, vol. 19, no. 11, Article ID 115604, 2008.

[7] R. Gunawidjaja, S. Peleshanko, H. Ko, and V. V. Tsukruk, "Bimetallic nanocobs: decorating silver nanowires with gold nanoparticles," Advanced Materials, vol. 20, no. 8, pp. 15441549, 2008.

[8] J. L. Seung, M. B. Jeong, and M. Moskovits, "Polarizationdependent surface-enhanced raman scattering from a silvernanoparticle-decorated single silver nanowire," Nano Letters, vol. 8, no. 10, pp. 3244-3247, 2008.

[9] H. Wei, F. Hao, Y. Huang, W. Wang, P. Nordlander, and $\mathrm{H}$. Xu, "Polarization dependence of surface-enhanced Raman scattering in gold nanoparticle-nanowire systems," Nano Letters, vol. 8, no. 8, pp. 2497-2502, 2008.

[10] B. J. Wiley, Y. Chen, J. M. McLellan et al., "Synthesis and optical properties of silver nanobars and nanorice," Nano Letters, vol. 7, no. 4, pp. 1032-1036, 2007.

[11] J. M. McLellan, Z. Y. Li, A. R. Siekkinen, and Y. Xia, “The SERS activity of a supported ag nanocube strongly depends on its orientation relative to laser polarization," Nano Letters, vol. 7, no. 4, pp. 1013-1017, 2007.

[12] H. Wang, D. W. Brandl, F. Le, P. Nordlander, and N. J. Halas, "Nanorice: a hybrid plasmonic nanostructure," Nano Letters, vol. 6, no. 4, pp. 827-832, 2006.

[13] H. Qi, D. Alexson, O. Glembocki, and S. M. Prokes, "Plasmonic coupling on dielectric nanowire core-metal sheath composites," Nanotechnology, vol. 21, no. 8, Article ID 085705, 2010.

[14] H. Qi, D. Alexson, O. Glembocki, and S. M. Prokes, "The effect of size and size distribution on the oxidation kinetics and plasmonics of nanoscale Ag particles," Nanotechnology, vol. 21, no. 21, Article ID 215706, 2010.

[15] K. Kneipp, H. Kneipp, R. Manoharan et al., "Extremely large enhancement factors in surface-enhanced Raman scattering for molecules on colloidal gold clusters," Applied Spectroscopy, vol. 52, no. 12, pp. 1493-1497, 1998.

[16] H. Xu, J. Aizpurua, M. Käll, and P. Apell, "Electromagnetic contributions to single-molecule sensitivity in surfaceenhanced Raman scattering," Physical Review E, vol. 62, no. 3, pp. 4318-4324, 2000.
[17] Z. Wang, S. Pan, T. D. Krauss, H. Du, and L. J. Rothberg, "The structural basis for giant enhancement enabling singlemolecule Raman scattering," Proceedings of the National Academy of Sciences of the United States of America, vol. 100, no. 15, pp. 8638-8643, 2003.

[18] K. Kneipp, Y. Wang, R. R. Dasari, and M. S. Feld, "Approach to single molecule detection using surface-enhanced resonance Raman scattering SERRS: a study using rhodamine 6G on colloidal silver," Applied Spectroscopy, vol. 49, no. 6, pp. 780784, 1995.

[19] D. M. Kuncicky, S. D. Christesen, and O. D. Velev, "Role of the micro- and nanostructure in the performance of surfaceenhanced Raman scattering substrates assembled from gold nanoparticles," Applied Spectroscopy, vol. 59, no. 4, pp. 401409, 2005.

[20] P. M. Tessier, O. D. Velev, A. T. Kalambur, J. F. Rabolt, A. M. Lenhoff, and E. W. Kaler, "Assembly of gold nanostructured films templated by colloidal crystals and use in surfaceenhanced Raman spectroscopy," Journal of the American Chemical Society, vol. 122, no. 39, pp. 9554-9555, 2000.

[21] A. Tao, F. Kim, C. Hess et al., "Langmuir-Blodgett silver nanowire monolayers for molecular sensing using surfaceenhanced Raman spectroscopy," Nano Letters, vol. 3, no. 9, pp. 1229-1233, 2003.

[22] S. M. Prokes, O. J. Glembocki, R. W. Rendell, and M. G. Ancona, "Enhanced plasmon coupling in crossed dielectric/metal nanowire composite geometries and applications to surface-enhanced Raman spectroscopy," Applied Physics Letters, vol. 90, no. 9, Article ID 093105, 3 pages, 2007.

[23] COMSOL Multiphysics, COMSOL Inc., http://www.comsol .com/.

[24] J. Zhao, A. O. Pinchuk, J. M. McMahon et al., "Methods for describing the electromagnetic properties of silver and gold nanoparticles," Accounts of Chemical Research, vol. 41, no. 12, pp. 1710-1720, 2008.

[25] M. Micic, N. Klymyshyn, Y. D. Suh, and H. P. Lu, "Finite element method simulation of the field distribution for AFM tip-enhanced surface-enhanced Raman scanning microscopy," Journal of Physical Chemistry B, vol. 107, no. 7, pp. 1574-1584, 2003.

[26] H. Ditlbacher, A. Hohenau, D. Wagner et al., "Silver nanowires as surface plasmon resonators," Physical Review Letters, vol. 95, no. 25, Article ID 257403, 4 pages, 2005.

[27] M. W. Knight and N. J. Halas, "Nanoshells to nanoeggs to nanocups: optical properties of reduced symmetry core-shell nanoparticles beyond the quasistatic limit," New Journal of Physics, vol. 10, Article ID 105006, 2008.

[28] M. W. Knight, Y. Wu, J. B. Lassiter, P. Nordlander, and N. J. Halas, "Substrates matter: influence of an adjacent dielectric on an individual plasmonic nanoparticle," Nano Letters, vol. 9, no. 5, pp. 2188-2192, 2009.

[29] T. Laroche and C. Girard, "Near-field optical properties of single plasmonic nanowires," Applied Physics Letters, vol. 89, no. 23, Article ID 233119, 3 pages, 2006.

[30] M. W. Knight, N. K. Grady, R. Bardhan, F. Hao, P. Nordlander, and N. J. Halas, "Nanoparticle-mediated coupling of light into a nanowire," Nano Letters, vol. 7, no. 8, pp. 2346-2350, 2007.

[31] C. Jiang, W. Y. Lio, and V. V. Tsukruk, "Surface enhanced raman scattering monitoring of chain alignment in freely suspended nanomembranes," Physical Review Letters, vol. 95, no. 11, Article ID 115503, 4 pages, 2005.

[32] J. Ding, V. I. Birss, and G. Liu, "Formation and properties of polystyrene-block-poly(2-cinnamoylethyl methacrylate) brushes studied by surface-enhanced raman scattering 
and transmission electron microscopy," Macromolecules, vol. 30, no. 5, pp. 1442-1448, 1997.

[33] W. M. Sears, J. L. Hunt, and J. R. Stevens, "Raman scattering from polymerizing styrene. I. Vibrational mode analysis," Journal of Chemical Physics, vol. 75, no. 4, pp. 1589-1598, 1981.

[34] V. Zucolotto, M. Ferreira, C. Cordeiro et al., "Unusual interactions binding iron tetrasulfonated phthalocyanine and poly(allylamine hydrochloride) in layer-by-layer films," Journal of Physical Chemistry B, vol. 107, no. 16, pp. 3733-3737, 2003.

[35] R. Aroca and A. Thedchanamoorthy, "Vibrational studies of molecular organization in evaporated phthalocyanine thin solid films," Chemistry of Materials, vol. 7, no. 1, pp. 69-74, 1995.

[36] J. H. Kim, T. Kang, S. M. Yoo, S. Y. Lee, B. Kim, and Y. K. Choi, "A well-ordered flower-like gold nanostructure for integrated sensors via surface-enhanced Raman scattering," Nanotechnology, vol. 20, no. 23, Article ID 235302, 2009.

[37] C. A. Szafranski, W. Tanner, P. E. Laibinis, and R. L. Garrell, "Surface-enhanced Raman spectroscopy of aromatic thiols and disulfides on gold electrodes," Langmuir, vol. 14, no. 13, pp. 3570-3579, 1998.

[38] M. Takahashi, M. Fujita, and M. Ito, "SERS application to some electroorganic reactions," Surface Science, vol. 158, no. 1-3, pp. 307-313, 1985.

[39] A. A. Mani, Z. D. Schultz, B. Champagne et al., "Molecule orientation in self-assembled monolayers determined by infrared-visible sum-frequency generation spectroscopy," Applied Surface Science, vol. 237, no. 1-4, pp. 444-449, 2004.

[40] K. T. Carron and L. G. Hurley, "Axial and azimuthal angle determination with surface-enhanced Raman spectroscopy: thiophenol on copper, silver, and gold metal surfaces," Journal of Physical Chemistry, vol. 95, no. 24, pp. 9979-9984, 1991.

[41] S. Frey, V. Stadler, K. Heister et al., "Structure of thioaromatic self-assembled monolayers on gold and silver," Langmuir, vol. 17, no. 8, pp. 2408-2415, 2001.

[42] L. J. Wan, M. Terashima, H. Noda, and M. Osawa, "Molecular orientation and ordered structure of benzenethiol adsorbed on gold(111)," Journal of Physical Chemistry B, vol. 104, no. 15, pp. 3563-3569, 2000.

[43] S. W. Han, S. J. Lee, and K. Kim, "Self-assembled monolayers of aromatic thiol and selenol on silver: comparative study of adsorptivity and stability," Langmuir, vol. 17, no. 22, pp. 69816987, 2001.

[44] A. Bilić, J. R. Reimers, and N. S. Hush, "The structure, energetics, and nature of the chemical bonding of phenylthiol adsorbed on the $\mathrm{Au}(111)$ surface: implications for densityfunctional calculations of molecular-electronic conduction," Journal of Chemical Physics, vol. 122, no. 9, Article ID 094708, 15 pages, 2005.

[45] C. J. Sandroff and D. R. Herschbach, "Surface-enhanced Raman study of organic sulfides adsorbed on silver: facile cleavage of S-S and C-S bonds," Journal of Physical Chemistry, vol. 86, no. 17, pp. 3277-3279, 1982.

[46] L. Gunnarsson, E. J. Bjerneld, H. Xu, S. Petronis, B. Kasemo, and M. Käll, "Interparticle coupling effects in nanofabricated substrates for surface-enhanced Raman scattering," Applied Physics Letters, vol. 78, no. 6, 3 pages, 2001.

[47] P. Mohanty, I. Yoon, T. Kang et al., "Simple vapor-phase synthesis of single-crystalline Ag nanowires and single-nanowire surface-enhanced raman scattering," Journal of the American Chemical Society, vol. 129, no. 31, pp. 9576-9577, 2007.
[48] M. Moskovits, "Surface-enhanced spectroscopy," Reviews of Modern Physics, vol. 57, no. 3, pp. 783-826, 1985.

[49] D. S. Wang and M. Kerker, "Enhanced Raman scattering by molecules adsorbed at the surface of colloidal spheroids," Physical Review B, vol. 24, no. 4, pp. 1777-1790, 1981.

[50] P. J. Kottman, O. J. F. Martin, D. R. Smith, and S. Schultz, "Plasmon resonances of silver nanowires with a nonregular cross section," Physical Review B, vol. 64, no. 23, Article ID 235402, 10 pages, 2001.

[51] R. T. Hill, J. J. Mock, Y. Urzhumov et al., "Leveraging nanoscale plasmonic modes to achieve reproducible enhancement of light," Nano Letters, vol. 10, no. 10, pp. 4150-4154, 2010.

[52] J. Chen, T. Mårtensson, K. A. Dick et al., "Surface-enhanced Raman scattering of rhodamine 6G on nanowire arrays decorated with gold nanoparticles," Nanotechnology, vol. 19, no. 27, Article ID 275712, 2008.

[53] R. Kattumenu, C. H. Lee, L. Tian, M. E. McConney, and S. Singamaneni, "Nanorod decorated nanowires as highly efficient SERS-active hybrids," Journal of Materials Chemistry, vol. 21, no. 39, pp. 15218-15223, 2011. 

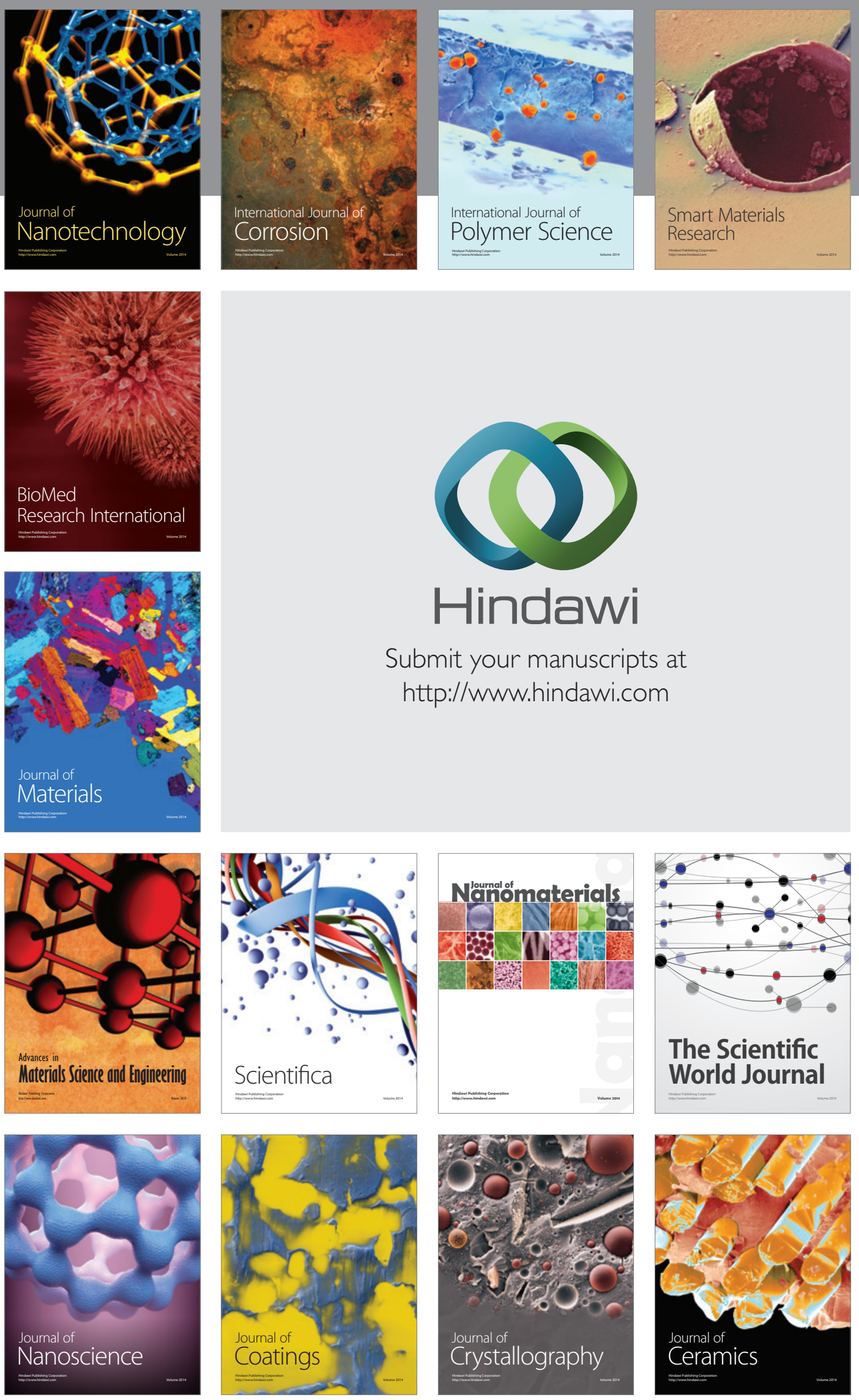

The Scientific World Journal

Submit your manuscripts at

http://www.hindawi.com

\section{World Journal}

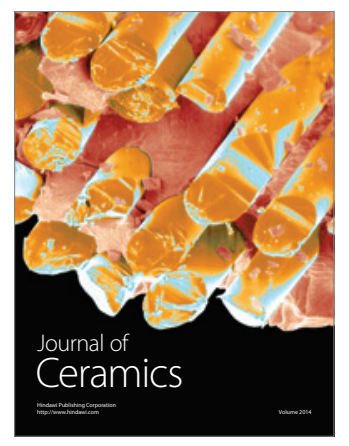

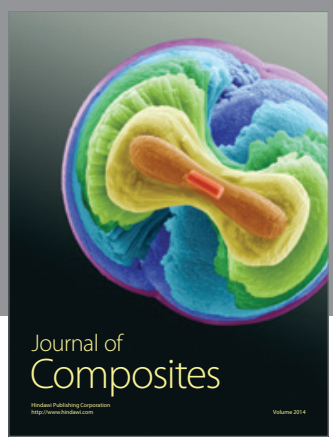
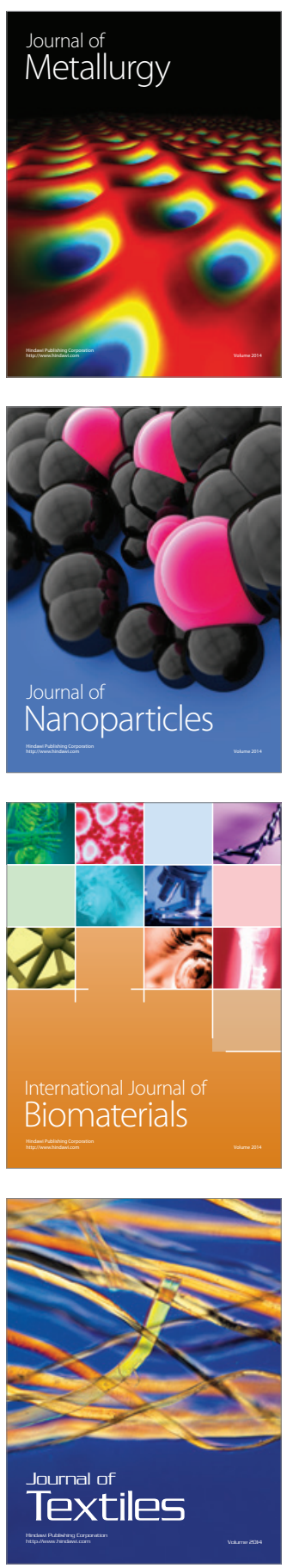